\title{
Inflammation, platelet activity, renal function and major cardiovascular events in patients with severe peripheral artery disease
}

\author{
Mislav Vrsalović*, Ksenija Vučur², Tomislav Krčmar², Boris Car ${ }^{2}$ \\ 'University of Zagreb School of Medicine, University Hospital Centre "Sestre milosrdnice", Zagreb, Croatia \\ ${ }^{2}$ University Hospital Centre "Sestre milosrdnice", Zagreb, Croatia
}

Objectives: Inflammatory markers and impaired renal function are the predictors of adverse outcome in patients with peripheral arterial disease (PAD). Despite interrelated pathophysiology between inflammation, platelets and renal function, these risk factors have not been examined together in the clinical setting. Therefore, the purpose of the study was to investigate their prognostic impact in PAD patients and to verify if they could add to the prognostic power of the ankle/brachial index (ABI).

Patients and Methods: The occurrence of major adverse cardiovascular events (MACE, defined as composite endpoint of death, myocardial infarction, stroke, percutaneous coronary intervention and coronary bypass surgery) was prospectively assessed in 174 PAD patients, Fontaine stages Ilb and III (66\% males, mean age 69.7 years, mean $\mathrm{ABI}$ 0.59). C-reactive protein (CRP), leukocyte count (WBC), mean platelet volume (MPV, an indicator of platelet reactivity) and estimated glomerular filtration rate (eGFR, calculated by MDRD formula) were obtained at the time of admission. Multivariate Cox regression analysis (adjusted for age, gender, traditional, cardiovascular risk factors, $A B I$, body mass index, concomitant coronary and carotid disease and medications used: statins, antiplatelets, antihypertensives) was applied to assess the predictive values of biomarkers on patients' outcome. Optimal cut-off points of WBC $\left(>7.3 \times 10^{\circ} / \mathrm{L}\right)$, MPV (>7.7 fL) and eGFR (<60 ml/min) to predict MACE were calculated with ROC analysis. Comparison between c-statistics was made to assess the better ability of biomarkers in combination with $\mathrm{ABI}$ vs. $\mathrm{ABI}$ alone to predict MACE in PAD patients.
Results: During a median follow-up of 24 months, 36 patients $(21 \%)$ had an event. Higher baseline levels of WBC, MPV and lower eGFR were significantly associated with future MACE by univariate analysis $(p<0.05)$, while CRP did not differ between the groups $(p=0.89)$. After multivariable analysis, WBC (HR 2.71, 95\% Cl 1.29-5.69), MPV (HR 2.89, $95 \% \mathrm{Cl} 1.35-6.18$ ) and eGFR (HR 2.12, 95\% Cl 1.05-4.25) remained significant independent predictors of cardiovascular events. The model that incorporated WBC, MPV and eGFR to $A B I$ improved the prediction of MACE; the area under ROC curve rose from 0.57 to 0.71 ( $p=0.02$ ).

Conclusion: Leukocyte count, mean platelet volume and impaired renal function are independently related to future cardiovascular events in severe PAD patients, and improved the prognostic value of the ankle/brachial index.

KEYWORDS: peripheral artery disease, markers, major adverse cardiovascular events.

CITATION: Cardiol Croat. 2013;8(9):317.

Received: $31^{\text {st }}$ Jul 2013

*Address for correspondence: KKlinički bolnički centar "Sestre milosrdnice", Vinogradska 29, HR-10000 Zagreb, Croatia.

Phone: +385-1-3787-111

E-mail: mislav.vrsalovic@zg.t-com.hr

\section{Literature}

1. Brevetti G, Giugliano G, Brevetti L, Hiatt WR. Inflammation in peripheral artery disease. Circulation. 2010;122(18):1862-75. 